\title{
Adaptive Online Parameter Estimation Algorithm of PEM Fuel Cells
}

\author{
Yashan Xing ${ }^{1,2}$, Jing $\mathrm{Na}^{2}$, Ramon Costa-Castelló ${ }^{1}$
}

\begin{abstract}
Since most of fuel cell models are generally nonlinearly parameterized functions, existing modeling techniques rely on the optimization approaches and impose heavy computational costs. In this paper, an adaptive online parameter estimation approach for PEM fuel cells is developed in order to directly estimate unknown parameters. The general framework of this approach is that the electrochemical model is first reformulated using Taylor series expansion. Then, one recently proposed adaptive parameter estimation method is further tailored to estimate the unknown parameters. In this method, the adaptive law is directly driven by the parameter estimation errors without using any predictors or observers. Moreover, parameter estimation errors can be guaranteed to achieve exponential convergence. Besides, the online validation of regressor matrix invertibility are avoided such that computation costs can be effectively reduced. Finally, comparative simulation results demonstrate that the proposed approach can achieve better performance than least square algorithm for estimating unknown parameters of fuel cells.
\end{abstract}

Index Terms-PEM Fuel Cell, nonlinearly parameterized system, online parameter estimation.

\section{INTRODUCTION}

Fuel cells have been considered a promising and environmental-friendly energy source in recent years [1]. Through the chemical reaction between hydrogen and oxygen, fuel cells can directly convert the chemical energy into water, electricity and heat [2]-[5]. Based on this chemical principle, there are various advantages of fuel cells, such as high efficiency, zero pollutant emission, and flexible installation, etc.

Extensive research has been carried out on modelling dynamic characteristics of fuel cells. On this topic, there exists three major ideas: i) physical models that are based on the material property, physical structure and chemical reaction, i.e., lumped models [6], [7], hierarchical models [8], threedimensional models [9]-[11]; ii) data-driven models which consider the fuel cell as a black box modelling by artificial intelligence-based approaches, i.e., Neural Network [12]; iii)

This work was partially funded by the Spanish national project MICAPEM (ref. DPI2015- 69286-C3-2-R, MINECO/FEDER) and the Spanish State Research Agency through the María de Maeztu Seal of Excellence to IRI (MDM-2016-0656). This work was partially supported by AGAUR of Generalitat de Catalunya through the Advanced Control Systems (SAC) group grant (2017 SGR 482) and Chinese Scholarship Council (CSC) under grant (201808390007). This work has been done with the partial support of ACCIÓ (Operational Program FEDER Catalunya 2014-2020) through the REFER project (COMRDI15-1-0036-11)

${ }^{1}$ Institut de Robòtica i Informàtica Industrial, CSIC-UPC Llorens i Artigas 4-6, 08028 Barcelona, Spain (Email: yashan.xing@upc.com,ramon.costa@upc.edu)

${ }^{2}$ Faculty of Mechanical \& Electrical Engineering, Kunming University of Science \& Technology, Kunming, 650500, P.R. China. (Email: najing25@163.com) semi-empirical models that combine some physical characteristics with partially empirical variables [13]. However, most of those models are complex, nonlinear and strongly coupled where it is very difficult to apply control techniques and optimization algorithms. Besides, some parameters in the fuel cell model are not practically feasible to directly measure, and some parameters can suddenly change when fuel cells operate under degradation condition. Thus, the parameter estimation approaches for fuel cells are very helpful to understand the operation process.

The lumped or semi-empirical models of fuel cells can be considered as a nonlinearly parameterized system, which is hard to directly apply well-recognized estimation approaches. In order to address this problem, some optimization techniques are used to estimation parameters of fuel cells. In [14], [15], recursive least square (LS) algorithms were proposed to estimate parameters in a semi-empirical model. However, these approaches assume that noise or disturbance is uncorrelated and independent of the regression vector. In [2], [16], genetic algorithm combined with curve fitting procedure was used to estimate fuel cell parameters. Nevertheless, there are still some drawbacks for genetic algorithm, such as low speed. Wenyin et al. [17] proposed an adaptive differential evolution algorithm to estimate parameters in the electrochemical model.

In this paper, we tailor the adaptive parameter estimation approach for a polymer electrolyte membrane (PEM) fuel cell and present a modified adaptive estimation method based on our recent work [18], [19], which proposed a novel adaptive parameter estimation framework for linearly parameterized systems. Since unknown parameters to be estimated are embedded in the nonlinear function of fuel cells, those parameters are first extracted by using Taylor series expansion. In this way, the nonlinearly parameterized system can be reformulated into a linearly parameterized system. Then the proposed adaptive law driven by parameter estimation errors is used to estimate unknown parameters in the electrochemicial model of the PEM fuel cells. The estimation error can be guaranteed exponential convergence under the persistent excitation condition. Moreover, the online validation of the invertibility of the regressor matrix can be avoided such that the online estimation and faster convergence are achieved. Compared to existing parameter estimation methods for fuel cell [14]-[17], the proposed adaptive parameter estimation approach does not need the recursive computation and avoids designing any observer/predictor. Finally, comparative simulations between the proposed method and the LS method demonstrate the estimation efficacy of the proposed method for fuel cells. 


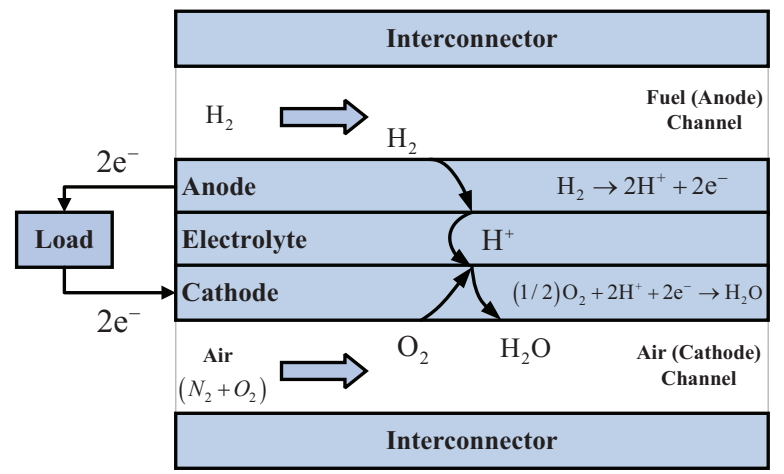

Fig. 1. Schematic diagram of the fuel cell

\section{FUEL CELL MODEL}

Fig. 1 depicts the open-cathode fuel cell that will be used in this work. Its modelling and experiment validation have been addressed in our earlier work [20], [21].

Before we introduce the model, the following assumptions are required:

Assumption 1: All gases are the ideal gasses.

Assumption 2: The temperature for the whole fuel cell is uniformly distributed.

Assumption 3: The heat capacity of gases is negligible.

The PEM fuel cell polarization curve can be written as [3]:

$$
v_{f c}=n_{c e l l} \cdot\left(E_{n e r}-v_{a c t}-v_{c o n}-v_{o h m}\right)
$$

where $n_{\text {cell }}$ is the number of cells in the fuel cell stack; $E_{n e r}$ is the Nernst voltage; $v_{a c t}$ represents the activation losses; $v_{c o n}$ is the concentration losses; and $v_{o h m}$ represents the ohmic losses.

The Nernst voltage can be approximately expressed as a function of gas partial pressure and temperature. In order to predict the net fuel cell voltage, $v_{f c}$, the Nernst voltage is thus very necessary to be estimated.

In practice, the open circuit potential of fuel cells is significantly lower than the theoretical potential voltage. There are three typical potential losses in the fuel cell which are activation losses, $v_{a c t}$, ohmic losses, $v_{o h m}$, concentration losses, $v_{c o n}$. Since concentration losses only happen due to the diffusion of reactants and products, it has less impact on potential losses. Thus, concentration losses are not considered in this paper.

The activation potential loss is the energy that reactants must overcome in the chemical reaction. It usually occurs at each electrode-electrolyte interface. The activation loss at the cathode channel is governed by the Tafel equation [22]:

$$
v_{a c t}=\frac{R T_{f c}}{\alpha n F} \cdot f\left(T_{f c}, i_{f c}\right)
$$

where $i_{f c}$ is the stack current; $T_{f c}$ is the stack temperature; $\alpha$ is the charge transfer coefficient; $R$ and $F$ represent gas constant and Faraday's constant, respectively. Function $f\left(T_{f c}, i_{f c}\right)$ is expressed as:

$$
f\left(T_{f c}, i_{f c}\right)=\ln \left(\frac{i_{f c}}{A_{c l} i_{0}^{A E C D}}\right)
$$

where $A_{c l}$ is the surface area of the catalyst layer. The apparent exchange current density $i_{0}^{A E C D}$ is obtained as:

$$
i_{0}^{A E C D}=i_{0}^{r e f} \cdot E C S A \cdot\left(\frac{P_{O_{2}}}{P_{r e f}}\right)^{0.5} e^{-\frac{E_{c a}}{R T_{f c}}\left(1-\frac{T_{f c}}{T_{r e f}}\right)}
$$

where $i_{0}^{r e f}$ is the initial exchange current density of cathode catalyst layer at the atmosphere condition $\left(T_{r e f}=25^{\circ} \mathrm{C}\right.$ and $P_{r e f}=1 \mathrm{~atm}$ ); $E_{c a}$ is activation energy in the cathode catalyst layer; ECSA represents the electrochemical active surface area; $P_{\mathrm{O}_{2}}$ is partial pressure of oxygen.

The ohmic loss is expressed as:

$$
v_{o h m}=r_{o h m} i_{f c}
$$

where $r_{o h m}$ is the ohmic resistance.

The thermal energy balance in the fuel cell can be expressed as:

$$
m_{f c} C_{p, f c} \frac{\mathrm{d} T_{f c}}{\mathrm{~d} t}=\dot{H}_{t o t}-\dot{H}_{c o o l}
$$

where $m_{f c}$ and $C_{p, f c}$ are the mass and the heat capacity of fuel cell, respectively. The total exothermic heat flow, $\dot{H}_{t o t}$, is made up of exothermic thermal heat flow and electrical power. Specifically, the exothermic thermal heat flow can be approximately calculated by thermoneutral voltage. And $\dot{H}_{\text {cool }}$ represents the heat transfer between the produced heat and the ambient air. Thus, the total exothermic heat flow and cool heat flow can be represented as follows:

$$
\begin{aligned}
\dot{H}_{t o t} & =n_{\text {cell }} E_{t h} i_{f c}-v_{f c} i_{f c} \\
\dot{H}_{\text {cool }} & =\rho_{a i r} A_{e f f} v_{a i r} C_{p, a i r}\left(T_{f c}-T_{r e f}\right)
\end{aligned}
$$

where $E_{t h}$ is the thermoneutral voltage; $\rho_{\text {air }}$ is air density; $A_{\text {eff }}$ represents the effective inlet area of cathode channel; $C_{p, a i r}$ is specific heat capacity of air; $v_{a i r}$ represents the velocity of air flow.

\section{PARAMETER ESTIMATION ALGORITHM}

In this section, some parameters of the electrochemical model are selected to be estimated. As analyzed in Section II, the Nernst voltage, $E_{n e r}$, is necessary to be estimated since it affects the net voltage of fuel cell. Moreover, the charge transfer coefficient, $\alpha$, in the activation loss is usually a empirical value. And the electrochemical active surface area, $E C S A$, highly depends on the changes of water saturation, which is very difficult to be measured in the experiment. Besides, the ohmic resistance, $r_{o h m}$, is possible to change under different operation condition. Thus, the parameters to be estimated in this paper are set as:

$$
\boldsymbol{\theta}=\left[\begin{array}{llll}
E_{n e r} & r_{o h m} & \frac{1}{\alpha} & \frac{1}{E C S A}
\end{array}\right]^{\mathrm{T}}
$$

From (1) to (4), the lumped electrochemical model is a highly nonlinear function. Especially, the unknown parameter, $\frac{1}{E C S A}$ (corresponding to $\boldsymbol{\theta}_{4}$ ), is embedded in the nonlinear function $f\left(T_{f c}, i_{f c}\right)$, which increases extra difficulties in the parameter estimation algorithm design.

Considered the nonlinear parameterized function $f\left(T_{f c}, i_{f c}\right)$, the electrochemical model (1) of PEM 
fuel cells can be reformulated in a general notation as follows:

$$
\mathbf{y}=\mathbf{f}(\mathbf{x}, \mathbf{u}, \boldsymbol{\theta})
$$

where $\mathbf{x} \in \mathbb{R}^{p \times 1}$ is the system state vector; $\mathbf{u} \in \mathbb{R}^{n \times 1}$ is the system input vector; $\mathbf{y} \in \mathbb{R}^{n \times 1}$ is the output vector; $\boldsymbol{\theta} \in$ $\mathbb{R}^{m \times 1}$ defines the vector of unknown constant parameters; $\mathbf{f}(\mathbf{x}, \mathbf{u}, \boldsymbol{\theta}) \in \mathbb{R}^{n \times 1}$ is a nonlinearly parameterized function where unknown parameters are embedded.

Our objective is to propose a parameter estimation algorithm which allows to estimate the unknown parameter, $\boldsymbol{\theta}$, through using the measurable input and output. For this purpose, the parameter estimation algorithm requires the following assumptions:

Assumption 4: The system states, $\mathbf{x}$, the system input, $\mathbf{u}$ are bounded and measurable. And the unknown parameters, $\boldsymbol{\theta}$, are also bounded constants.

Assumption 5: The nonlinear function $\mathbf{f}(\mathbf{x}, \mathbf{u}, \boldsymbol{\theta})$ is a continuously second-order differentiable function with respect to $\theta$.

In order to extract the unknown parameter $\boldsymbol{\theta}$ from the nonlinear function $\mathbf{f}(\mathbf{x}, \mathbf{u}, \boldsymbol{\theta})$, the Taylor series expansion is performed. Thus, the nonlinear system (10) is reformulated as

$$
\mathbf{y}=\mathbf{f}(\mathbf{x}, \mathbf{u}, \hat{\boldsymbol{\theta}})+\sum_{i=1}^{m} \tilde{\theta}_{i} \frac{\partial}{\partial \theta_{i}} \mathbf{f}(\mathbf{x}, \mathbf{u}, \hat{\boldsymbol{\theta}})+\boldsymbol{\delta}(\tilde{\boldsymbol{\theta}})
$$

where $\tilde{\boldsymbol{\theta}}=\boldsymbol{\theta}-\hat{\boldsymbol{\theta}}$ is the estimated parameter error; $\hat{\boldsymbol{\theta}}$ represents the estimated parameter; $\boldsymbol{\delta}(\tilde{\boldsymbol{\theta}})$ is the Lagrange remainder term of the Taylor series expansion, which is considered as a bounded disturbance.

To facilitate the parameter estimation algorithm, the regressor matrix is defined and then the system of (11) can be represented as:

$$
\mathbf{y}=\boldsymbol{\Phi}(\mathbf{x}, \mathbf{u}, \hat{\boldsymbol{\theta}}) \boldsymbol{\theta}+\boldsymbol{\varphi}(\mathbf{x}, \mathbf{u}, \hat{\boldsymbol{\theta}})+\boldsymbol{\delta}(\tilde{\boldsymbol{\theta}})
$$

where

$$
\boldsymbol{\Phi}(\mathbf{x}, \mathbf{u}, \hat{\boldsymbol{\theta}})=\left[\begin{array}{ccc}
\left.\frac{\partial f_{1}(\mathbf{x}, \mathbf{u}, \boldsymbol{\theta})}{\partial \theta_{1}}\right|_{\boldsymbol{\theta}=\hat{\boldsymbol{\theta}}} & \cdots & \left.\frac{\partial f_{1}(\mathbf{x}, \mathbf{u}, \boldsymbol{\theta})}{\partial \theta_{m}}\right|_{\boldsymbol{\theta}=\hat{\boldsymbol{\theta}}} \\
\vdots & \ddots & \vdots \\
\left.\frac{\partial f_{n}(\mathbf{x}, \mathbf{u}, \boldsymbol{\theta})}{\partial \theta_{1}}\right|_{\boldsymbol{\theta}=\hat{\boldsymbol{\theta}}} & \cdots & \left.\frac{\partial f_{n}(\mathbf{x}, \mathbf{u}, \boldsymbol{\theta})}{\partial \theta_{m}}\right|_{\boldsymbol{\theta}=\hat{\boldsymbol{\theta}}}
\end{array}\right] \in \mathbb{R}^{n \times m}
$$

is the known regressor matrix which is based on inputs $\mathbf{u}$ and states $\mathbf{x} ; \boldsymbol{\varphi}(\mathbf{x}, \mathbf{u}, \hat{\boldsymbol{\theta}})=\mathbf{f}(\mathbf{x}, \mathbf{u}, \hat{\boldsymbol{\theta}})-\mathbf{\Phi}(\mathbf{x}, \mathbf{u}, \hat{\boldsymbol{\theta}}) \hat{\boldsymbol{\theta}}$ can be updated online.

Remark 1: Applying the first order Taylor series expansion, the unknown parameters, $\boldsymbol{\theta}$, are extracted from the nonlinear function $\mathbf{f}(\mathbf{x}, \mathbf{u}, \boldsymbol{\theta})$. The high-order term of Taylor series expansion $\boldsymbol{\delta}(\tilde{\boldsymbol{\theta}})$ will be vanished when the convergence of parameter estimation is achieved.

Following the process above, we will use the Taylor series expansion to extract the parameter $\boldsymbol{\theta}_{4}$. For brevity, we first define that

$$
\begin{gathered}
f_{1}=\frac{n_{c e l l} R T_{f c}}{n F} \\
f_{2}=\frac{i_{f c}}{A_{c l} i_{0}^{r e f}\left(\frac{P_{O_{2}}}{P_{\text {ref }}}\right)^{0.5} e^{-\frac{E_{c a}}{R T_{f c}}\left(1-\frac{T_{f c}}{T_{r e f}}\right)}}
\end{gathered}
$$

The term $f_{1} \theta_{3} \ln \left(f_{2} \hat{\theta}_{4}\right)$ is added and subtracted in the completed form of the lumped electrochemical model from (1) to (5). Then the Taylor series expansion is used to extract $\theta_{4}$. Thus, the regressor matrix is represented as:

$$
\boldsymbol{\Phi}=\left[\begin{array}{llll}
n_{\text {cell }} & -n_{\text {cell }} i_{f c} & -f_{1} \cdot \ln \left(f_{2} \hat{\theta}_{4}\right) & -f_{1} \cdot \frac{\hat{\theta}_{3}}{\left|\hat{\theta}_{4}\right|}
\end{array}\right]
$$

And the residual term in (12) is derived as: $\varphi=f_{1} \hat{\theta}_{3}$.

Before developing the parameter estimation approach, the maximum and minimum matrix eigenvalues are denoted as $\lambda_{\min }\{\cdot\}, \lambda_{\max }\{\cdot\}$ for brevity.

In order to estimate unknown parameters, auxiliary matrix $\mathbf{P} \in \mathbb{R}^{m \times m}$ and vector $\mathbf{q} \in \mathbb{R}^{m \times 1}$ are defined as:

$$
\begin{cases}\dot{\mathbf{P}}=-\ell \mathbf{P}+\boldsymbol{\Phi}^{\mathrm{T}} \boldsymbol{\Phi}, & \mathbf{P}(0)=0 \\ \dot{\mathbf{q}}=-\ell \mathbf{q}+\boldsymbol{\Phi}^{\mathrm{T}}(\mathbf{y}-\boldsymbol{\varphi}(\mathbf{x}, \mathbf{u}, \hat{\boldsymbol{\theta}})), & \mathbf{q}(0)=0\end{cases}
$$

where $\ell>0$ is a constant value. It can considered as a forgetting factor which guarantees the boundness of $\mathbf{P}$ and q.

Lemma 1 ( [18], [19]): If the regressor matrix $\boldsymbol{\Phi}(\mathbf{x}, \mathbf{u}, \hat{\boldsymbol{\theta}})$ satisfies the persistent excitation $(\mathrm{PE})$ condition (when $T>0$ and $\varepsilon>0, \int_{t}^{t+T} \boldsymbol{\Phi}^{\mathrm{T}}(\tau) \boldsymbol{\Phi}(\tau) \mathrm{d} \tau \geq \varepsilon I, \forall t \geq$ $0)$, the matrix $\mathbf{P}(t)$ defined in (14) is positive definite $\left(\lambda_{\min }(\mathbf{P}(t))>\rho>0, \forall t>0\right)$.

Proof: A detailed proof of the above lemma can be found in our previous work [18], [19], and thus it will not be presented here.

Then another auxiliary vector $\mathbf{w} \in \mathbb{R}^{m \times 1}$ can be defined as:

$$
\mathbf{w}=\mathbf{P} \hat{\boldsymbol{\theta}}-\mathbf{q}
$$

Lemma 2: From the auxiliary vector $\mathbf{w}$ given in (15), we can obtain that

$$
\mathbf{w}=-\mathbf{P} \tilde{\boldsymbol{\theta}}+\boldsymbol{\psi}
$$

where $\boldsymbol{\psi}(t)=-\int_{0}^{t} e^{-\ell(t-\tau)} \Phi^{\mathrm{T}}(\tau) \boldsymbol{\delta}(\tau) \mathrm{d} \tau$ is a bounded residual error, since $\delta$ is bounded, such that $\|\boldsymbol{\psi}\| \leq$ $\|\boldsymbol{\Phi}\|\|\boldsymbol{\delta}\| / \ell=\mu, \forall \mu>0$.

Proof: In order to prove Lemma 2, the equation (14) can be derived as:

$$
\left\{\begin{array}{l}
\mathbf{P}(t)=\int_{0}^{t} e^{-\ell(t-\tau)} \boldsymbol{\Phi}^{\mathrm{T}}(\tau) \boldsymbol{\Phi}(\tau) \mathrm{d} \tau \\
\mathbf{q}(t)=\int_{0}^{t} e^{-\ell(t-\tau)} \boldsymbol{\Phi}^{\mathrm{T}}(\tau)(\mathbf{y}-\boldsymbol{\varphi}(x, u, \hat{\theta})) \mathrm{d} \tau
\end{array}\right.
$$

From (12), we can reformulate that $\mathbf{y}-\boldsymbol{\varphi}=\boldsymbol{\Phi} \boldsymbol{\theta}+\boldsymbol{\delta}$ and then verify that $\mathbf{q}=\mathbf{P} \boldsymbol{\theta}+\boldsymbol{\psi}$, which are substituted into (15). Finally, the equation (16) can be derived and proved.

The adaptive law for updating $\hat{\boldsymbol{\theta}}$ is defined as:

$$
\dot{\hat{\boldsymbol{\theta}}}=-\Gamma \mathbf{w}
$$

where $\Gamma>0$ is a constant diagonal matrix.

Theorem 1: Considering system (12) with unknown parameter $\boldsymbol{\theta}$, the adaptive law (18) is used. If the regressor matrix $\Phi$ is satisfied PE condition, then the estimation error $\tilde{\boldsymbol{\theta}}$ converges to a small compact set given by:

$$
\|\tilde{\boldsymbol{\theta}}\| \leq \sqrt{\frac{2 \beta / \eta+e^{-\eta t} \lambda_{\max }\left\{\boldsymbol{\Gamma}^{-1}\right\}\|\tilde{\theta}(0)\|^{2}}{\lambda_{\min }\left\{\boldsymbol{\Gamma}^{-1}\right\}}}
$$


where the matrix $\mathbf{P}$ is positive definite $\left(\lambda_{\min }(\mathbf{P})>\rho>\right.$ $0, \forall t>0)$; the residual error is bounded $(\|\boldsymbol{\psi}\| \leq \mu)$; and $n>1 / 2 \rho$ is a positive constant.

Proof: The Lyapunov function is considered as

$$
V=\frac{1}{2} \tilde{\boldsymbol{\theta}}^{\mathrm{T}} \boldsymbol{\Gamma}^{-1} \tilde{\boldsymbol{\theta}}
$$

Then we can calculate the derivation of (19) along (16) and (18) as

$$
\dot{V}=\tilde{\boldsymbol{\theta}}^{\mathrm{T}} \boldsymbol{\Gamma}^{-1} \dot{\tilde{\boldsymbol{\theta}}}=-\tilde{\boldsymbol{\theta}}^{\mathrm{T}} \boldsymbol{\Gamma}^{-1} \dot{\hat{\boldsymbol{\theta}}}=\tilde{\boldsymbol{\theta}}^{\mathrm{T}} \mathbf{w}=\tilde{\boldsymbol{\theta}}^{\mathrm{T}}(-\boldsymbol{P} \tilde{\boldsymbol{\theta}}+\Psi)
$$

By applying Young's inequality $a^{\mathrm{T}} b \leq a^{\mathrm{T}} a / 2 n+n b^{\mathrm{T}} b / 2$ with a positive constant $n>0$, we can further derive that

$$
\begin{aligned}
\dot{V} & \leq-\rho\|\tilde{\boldsymbol{\theta}}\|^{2}+\tilde{\boldsymbol{\theta}}^{\mathrm{T}} \boldsymbol{\Psi} \leq-\left(\rho-\frac{1}{2 n}\right)\|\tilde{\boldsymbol{\theta}}\|^{2}+\frac{n\|\boldsymbol{\Psi}\|^{2}}{2} \\
& \leq-\eta V+\beta
\end{aligned}
$$

where $\eta=2(\rho-1 / 2 n) / \lambda_{\max }\left\{\boldsymbol{\Gamma}^{-1}\right\}, \beta=n \mu^{2} / 2$ both are positive constants for $n>1 / 2 \rho$. Moreover, the solution of (21) is $V(t) \leq e^{-\eta t} V(0)+\beta / \eta$. Based on the definition of (19), it can be derived that $\|\tilde{\boldsymbol{\theta}}\| \leq \sqrt{2 V(t) / \lambda_{\min }\left\{\boldsymbol{\Gamma}^{-1}\right\}} \leq$ $\sqrt{2\left(\beta / \eta+V(0) e^{-\eta t}\right) / \lambda_{\min }\left\{\boldsymbol{\Gamma}^{-1}\right\}}$. Therefore, the estimation error $\tilde{\boldsymbol{\theta}}$ will exponentially converge to a small compact set as defined in Theorem 1.

Remark 2: The ultimate size of estimation errors $\tilde{\boldsymbol{\theta}}$ depends on the excitation level $\rho$ and the learning gain $\Gamma$ as shown in Theorem 1. A higher excitation level $\rho$ and a higher gain $\boldsymbol{\Gamma}$ can increase the convergence speed. Moreover, a larger filter constant $\ell$ can reduce the residual error as shown in Lemma 2. However, the larger filter constant $\ell$ may produce a larger DC gain, and then reduce the amplitude of the learing gain $\Gamma$ so as to decrease the convergence rate. Thus, the filter constant $\ell$ should be chosen small practically.

Remark 3: From Lemma 2, the estimation error $\mathbf{P} \tilde{\boldsymbol{\theta}}$ involved in the auxiliary matrix $\mathbf{w}$ is used to drive the new adaptive law (18) and thus there is no need to use observers in the classical parameter estimation scheme.

Remark 4: The sufficient condition to prove the convergence of the proposed adaptive law (18) is that the regressor matrix $\boldsymbol{\Phi}$ satisfies the PE condition. However, there is still an open problem to online validate the PE condition. In this paper, we provide a feasible method to online test this condition for the proposed parameter estimation framework, as shown in Lemma 1.

\section{THE LEAST SQUARE ALGORITHM}

In this section the least square (LS) algorithm with a variable forgetting factor [23] is represented.

The tracking error $\mathbf{e}$ is calculated as follows:

$$
\mathbf{e}=\mathbf{y}-\hat{\mathbf{y}}=\mathbf{y}-\boldsymbol{\Phi} \hat{\boldsymbol{\theta}}-\boldsymbol{\varphi}=\boldsymbol{\delta}(\tilde{\boldsymbol{\theta}})+\boldsymbol{\Phi} \tilde{\boldsymbol{\theta}} .
$$

For LS method with variable forgetting factor [23], the adaptive law is driven by the tracking error $\mathbf{e}$ as follows:

$$
\dot{\hat{\boldsymbol{\theta}}}=\frac{\mathbf{H} \boldsymbol{\Phi}^{\mathrm{T}} \mathbf{e}}{m^{2}}
$$

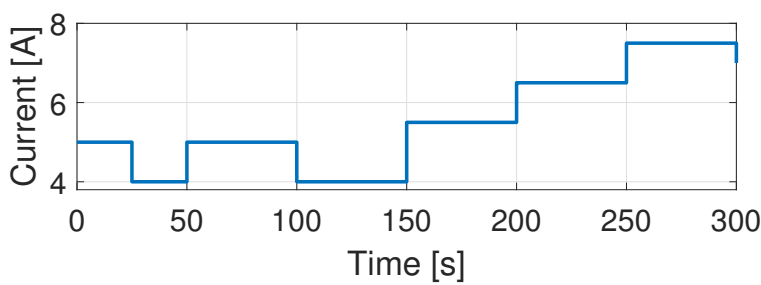

Fig. 2. The input current of fuel cell stack without disturbance

TABLE I

Fuel Cell Model Parameters

\begin{tabular}{ll}
\hline Parameter & Value \\
\hline \hline$A_{c l}$, surface area of catalyst layer & $2.5 \times 10^{-3}\left[\mathrm{~m}^{2}\right]$ \\
$A_{e f f}$, effective area of cathode channel & $8.5 \times 10^{-3}\left[\mathrm{~m}^{2}\right]$ \\
$C_{p, \text { air }}$, heat capacity of air & $1005\left[\mathrm{~J} \cdot \mathrm{kg}^{-1} \cdot \mathrm{K}^{-1}\right]$ \\
$C_{p, f c}$, heat capacity of stack & $1200\left[\mathrm{~J} \cdot \mathrm{kg}{ }^{-1} \cdot \mathrm{K}^{-1}\right]$ \\
$E_{c a}$, activation energy of cathode channel & $7 \times 10^{4}\left[\mathrm{~J} \cdot \mathrm{mol}^{-1}\right]$ \\
$E C S A$, electrochemical active surface area & \\
$E_{n e r}$, Nernst voltage & $1.16[\mathrm{~V}]$ \\
$E_{t h}$, thermoneutral voltage & $1.23[\mathrm{~V}]$ \\
$F$, Faraday's constant & $96485\left[\mathrm{C} \cdot \mathrm{mol}^{-1}\right]$ \\
$i_{0}^{r e f}$, exchange current density & $5 \times 10^{-3}\left[\mathrm{~A} \cdot \mathrm{m}^{-2}\right]$ \\
$m{ }_{f c}$, stack mass & $0.3[\mathrm{~kg}]$ \\
$n$, number of electrons & 2 \\
$n_{c e l l}$, number of cells & 24 \\
$P_{O_{2}}$, partial pressure of oxygen & $2.1 \times 10^{4}[\mathrm{~Pa}]$ \\
$P_{r e f}$, atmosphere pressure & $10^{5}[\mathrm{~Pa}]$ \\
$r_{\text {ohm }}$, ohmic resistance & $0.7[\Omega]$ \\
$R$, gas constant & $8.314\left[\mathrm{~J} \cdot \mathrm{mol}{ }^{-1} \cdot \mathrm{K}^{-1}\right]$ \\
$T_{r e f}$, atmosphere temperature & $298[\mathrm{~K}]$ \\
$v_{a i r}$, input air velocity & {$[\mathrm{m} \cdot \mathrm{s}-1]$} \\
$\alpha$, charge transfer coefficient & 0.28 \\
$\theta$, effective contact angle & $91\left[{ }^{\circ} \mathrm{C}\right]$ \\
$\rho_{\text {air }}$, air density & $1.205\left[\mathrm{~kg} \cdot \mathrm{m}^{3}\right]$ \\
\hline & \\
\hline &
\end{tabular}

where $m^{2}=1+\Phi^{\mathrm{T}} \Phi$ is a normalizing factor; $\mathbf{H}$ is a learning gain which is updated as:

$$
\dot{\mathbf{H}}=-\frac{\mathbf{H} \boldsymbol{\Phi}^{\mathrm{T}} \boldsymbol{\Phi} \mathbf{H}}{m^{2}}+\mathbf{K}\left(\mathbf{I}-\alpha_{1} \mathbf{H}\right) \mathbf{H}
$$

where the boundness of initial learning gain is manually defined as $0<\frac{1}{\chi} I \leq H(0)=\mathbf{H}_{\mathbf{0}}=\mathbf{H}_{\mathbf{0}}^{\mathrm{T}}<\frac{1}{\alpha_{1}} I$; and $\mathbf{K}>0$ is a constant diagonal matrix. Thus, the estimation error of LS algorithm is derived as:

$$
\dot{\tilde{\boldsymbol{\theta}}}=-\dot{\hat{\boldsymbol{\theta}}}=-\frac{\mathbf{H} \boldsymbol{\Phi}^{\mathrm{T}} \mathbf{e}}{m^{2}}
$$

In (22), the observer error e includes estimation errors $\tilde{\boldsymbol{\theta}}$ and the Lagrange remainder term from Taylor series expansion. Thus, the boundness of estimated parameter error may depend on the amplitude of this remainder term. Moreover, the LS algorithm have a potential possibility in producing the drifting and bursting phenomena. Besides, the convergence of estimation error could not be claimed without online verifying the $\mathrm{PE}$ condition even though the estimation error converges to zero.

\section{Simulation RESUlts}

In this section, the proposed parameter estimation method is validated by simulations using the proposed fuel cell model. In practice, the fan is used to remove the heat of fuel cell and simultaneously increase the amount of oxygen in the cathode channel. Thus, the air velocity can be fixed adjusting 

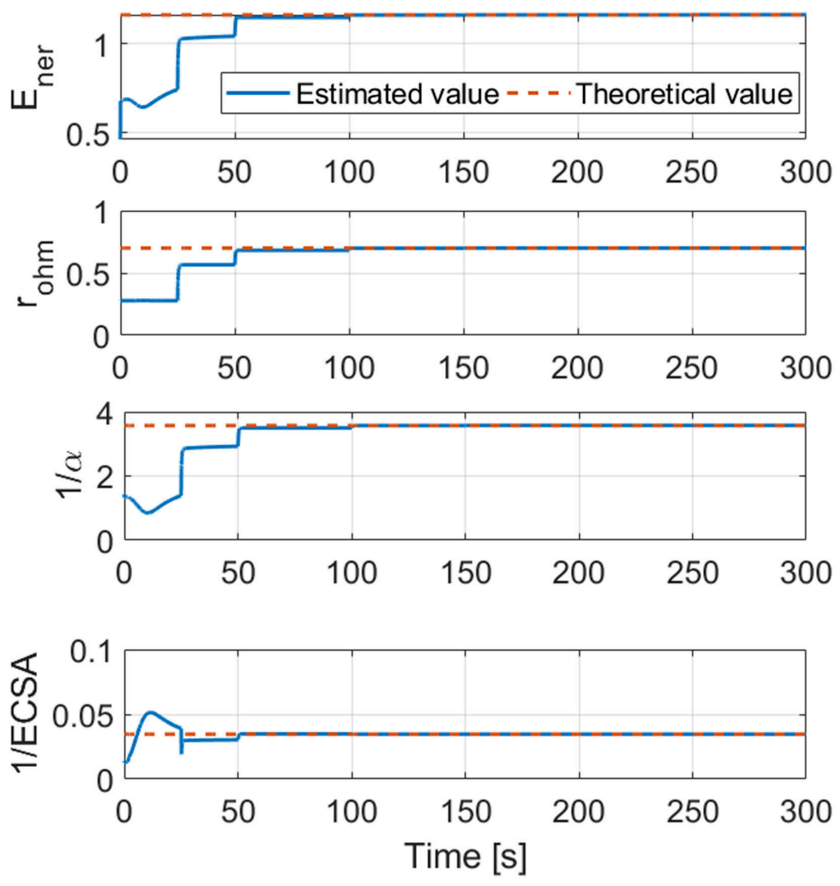

Fig. 3. Proposed method parameter estimation results.

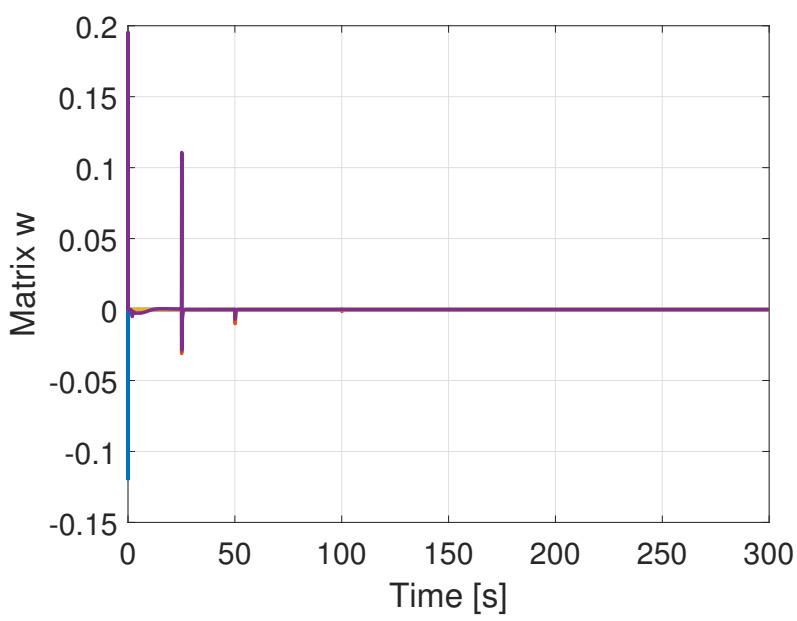

Fig. 4. The auxiliary matrix $\mathbf{w}$ evolution in the proposed method

the pulse-width-modulation (PWM) duty cycle signal of a fan [20]. The value of PWM duty cycle is $90 \%$ in this simulation. Moreover, the physical parameters of fuel cell are set as the same with the H-100 fuel cell parameters in [20], [21], which are summarized in TABLE I. The stack current are set as depicted in Fig. 2.

The parameters used in (14) and (18) are set as $\ell=3$, $\boldsymbol{\Gamma}=\operatorname{diag}\left(\left[\begin{array}{llll}750 & 36 & 2400 & 2.15\end{array}\right]\right)$. Due to the parameter $\theta_{4}$ in the natural logarithm, the initial value of $\theta_{4}$ cannot equal to zero. Thus, the initial values of unknown parameters are set as $\boldsymbol{\theta}_{\mathbf{0}}=\left[\begin{array}{llll}0.464 & 0.28 & 1.4286 & 0.0138\end{array}\right]^{\mathrm{t}}$.

Simulation results of the estimated parameter profiles are depicted in Fig. 3. It is shown that the estimated parameters converge to their true values and the adaptive law can achieve accurate estimation. The auxiliary matrix $\mathbf{w}$ converges to

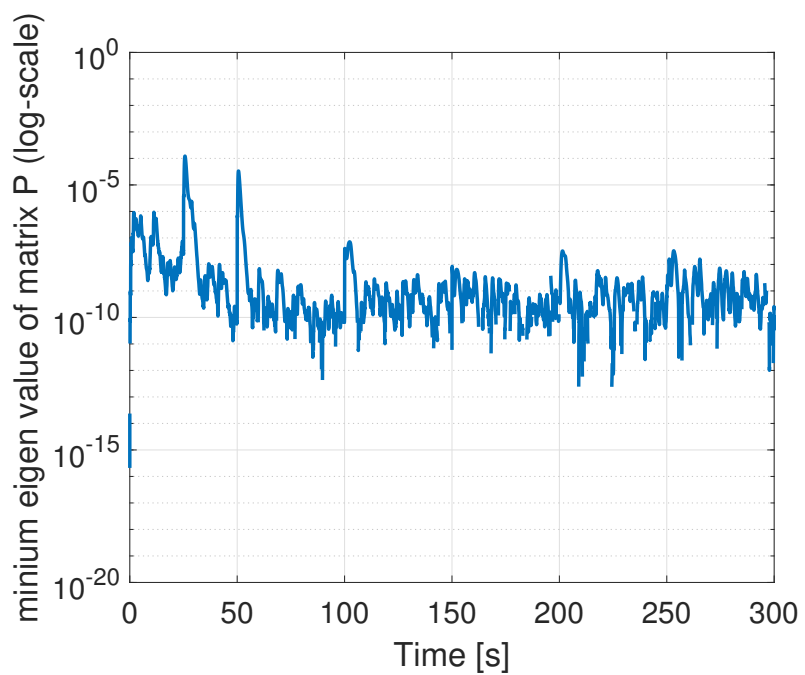

Fig. 5. Minimum eigenvalue of matrix $\mathbf{P}(\mathbf{t})$

zero as depicted in Fig. 4. In practice, we can online check whether the auxiliary matrix $\mathbf{w}$ converges to zero in order to make sure that the estimated parameters converge to the true value. Moreover, Fig. 5 illustrates that the minimum eigenvalue of matrix $\mathbf{P}$ is larger than zero such that the regressor vector satisfies the PE condition. This provides a feasible method to online test the PE condition.

In order to further reveal the merit of the proposed approach driven by the estimation error, the LS algorithm (23) with a variable forgetting factor (24) in Section IV is used to estimate the unknown parameters $\boldsymbol{\theta}$. The parameters used in (23) and (24) are set as $\mathbf{K}=$ $\operatorname{diag}\left(\left[\begin{array}{llll}9000 & 2500 & 6000 & 2\end{array}\right]\right), \chi=1$ and $\alpha_{1}=10^{-5}$.

Fig. 6 shows the parameter estimation results of LS method in (23) and (24). And the reconstructed output by the estimated results, the input of current and air velocity are depicted in Fig. 7. It is shown that the unknown parameters cannot be accurately estimated by LS method even though the observer errors converges to zero. Hence, the proposed adaptive method (18) can achieve better estimation results than the LS method (23).

\section{CONCLusions}

In this paper, an online parameter estimation approach for a nonlinearly parameterized system of fuel cells has been developed. The nonlinearly parameterized system is reformulated by the Taylor series expansion. Then, one recently developed adaptive parameter estimation method is tailored to achieve parameter estimation for fuel cells. The adaptive law in this method is directly driven by the parameter estimation errors. Moreover, this new estimation framework provides a feasible method to online test the required PE condition to guarantee the error convergence. The convergence of the estimation error has been proved in terms of Lyapunov method. Comparative simulation results illustrate the efficacy of the proposed estimation parameter method. Future work will focus on extending the proposed parameter estimation approach to the unknown time-varying parameters in the nonlinear functions of fuel cell systems. 

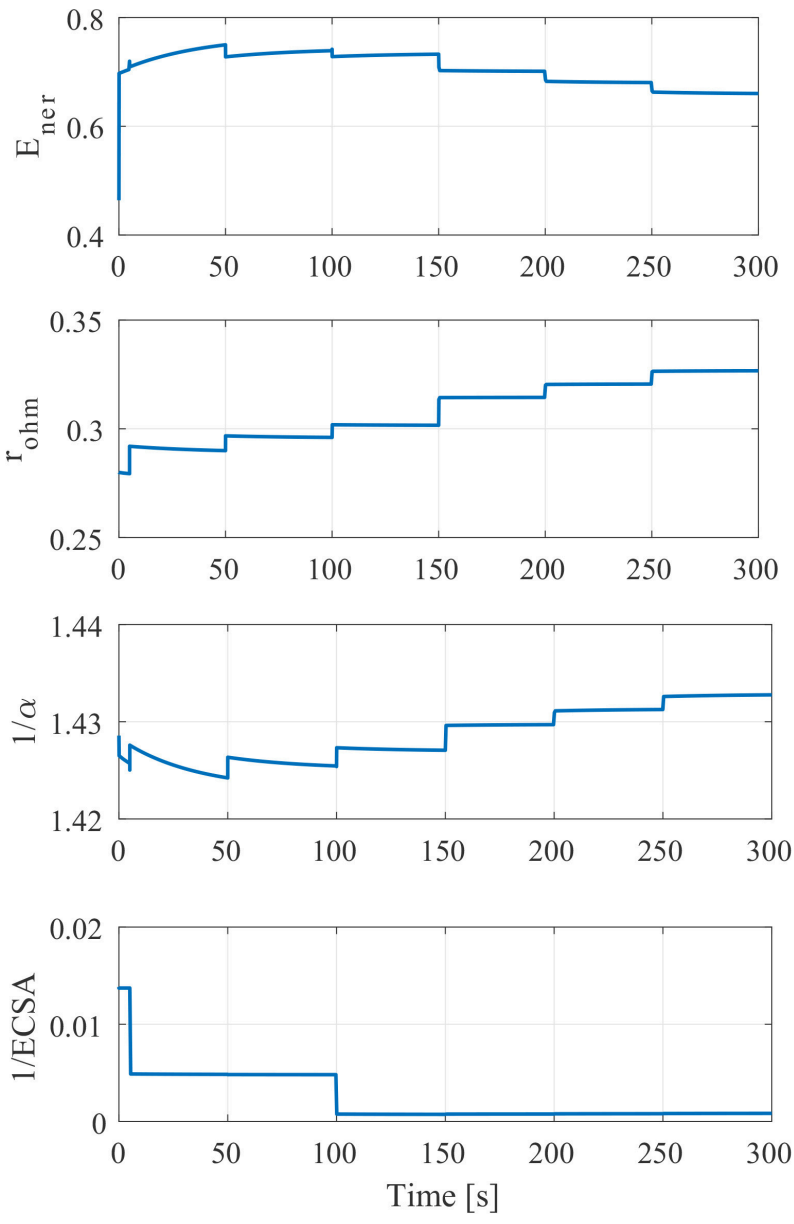

Fig. 6. Parameter estimation results of LS method

\section{REFERENCES}

[1] O. Z. Sharaf and M. F. Orhan, "An overview of fuel cell technology: Fundamentals and applications," Renewable and Sustainable Energy Reviews, vol. 32, pp. 810-853, 42014.

[2] E. Ariza, A. Correcher, C. Sánchez, A. Navarro-Pérez, and E. Garcia, "Thermal and electrical parameter identification of a proton exchange membrane fuel cell using genetic algorithm," Energies, vol. 11, p. 2099, 082018.

[3] F. Barbir, PEM Fuel Cells: Theory and Practice. Academic Press, 2005.

[4] P. Pei and H. Chen, "Main factors affecting the lifetime of Proton Exchange Membrane fuel cells in vehicle applications: A review," Applied Energy, vol. 125, pp. 60-75, 72014.

[5] P. Moçoteguy and A. Brisse, "A review and comprehensive analysis of degradation mechanisms of solid oxide electrolysis cells," International Journal of Hydrogen Energy, vol. 38, no. 36, pp. 15 887-15902, 122013.

[6] J. T. Pukrushpan, H. Peng, and A. G. Stefanopoulou, "ControlOriented Modeling and Analysis for Automotive Fuel Cell Systems," Journal of Dynamic Systems, Measurement, and Control, vol. 126, no. 1, p. 14, 32004.

[7] A. M. Murshed, B. Huang, and K. Nandakumar, "Control relevant modeling of planer solid oxide fuel cell system," Journal of Power Sources, vol. 163, no. 2, pp. 830-845, 12007.

[8] M. Sorrentino, C. Pianese, and Y. G. Guezennec, "A hierarchical modeling approach to the simulation and control of planar solid oxide fuel cells," Journal of Power Sources, vol. 180, no. 1, pp. 380-392, 5 2008.

[9] M. Hu, A. Gu, M. Wang, X. Zhu, and L. Yu, "Three dimensional, two phase flow mathematical model for PEM fuel cell: Part I. Model development," Energy Conversion and Management, vol. 45, no. 1112, pp. 1861-1882, 72004.

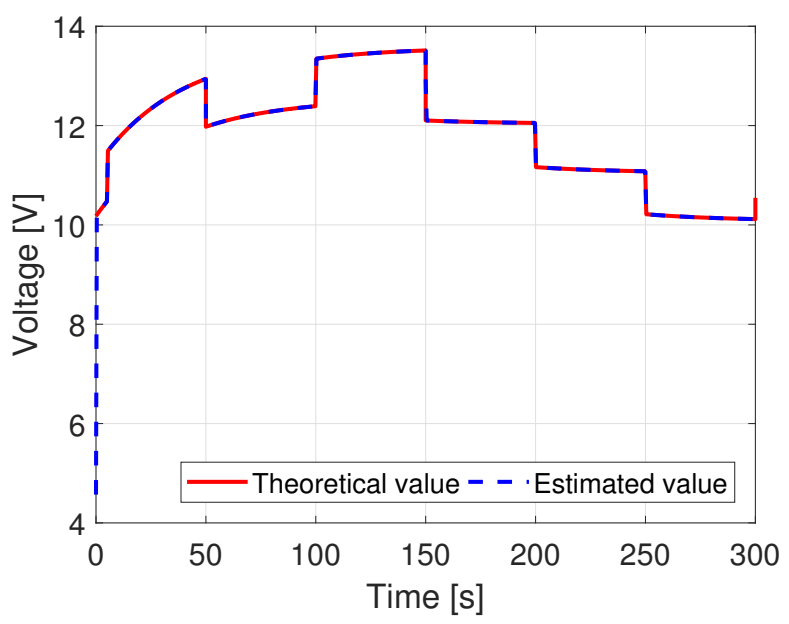

Fig. 7. Reconstructed output of LS method

[10] T. Berning and N. Djilali, "Three-dimensional computational analysis of transport phenomena in a PEM fuel cell-a parametric study,' Journal of Power Sources, vol. 124, no. 2, pp. 440-452, 112003.

[11] B. Haberman and J. Young, "Three-dimensional simulation of chemically reacting gas flows in the porous support structure of an integrated-planar solid oxide fuel cell," International Journal of Heat and Mass Transfer, vol. 47, no. 17-18, pp. 3617-3629, 82004.

[12] G. Napoli, M. Ferraro, F. Sergi, G. Brunaccini, and V. Antonucci, "Data driven models for a PEM fuel cell stack performance prediction," International Journal of Hydrogen Energy, vol. 38, no. 26, pp. $11628-11638,82013$.

[13] M. A. S. Al-Baghdadi, "Modelling of proton exchange membrane fuel cell performance based on semi-empirical equations," Renewable Energy, vol. 30, no. 10, pp. 1587-1599, 82005.

[14] K. Ettihir, L. Boulon, M. Becherif, K. Agbossou, and H. Ramadan, "Online identification of semi-empirical model parameters for PEMFCs," International Journal of Hydrogen Energy, vol. 39, no. 36, pp. 21 165-21 176, 122014.

[15] M. Kandidayeni, A. Macias, A. A. Amamou, L. Boulon, and S. Kelouwani, "Comparative Analysis of Two Online Identification Algorithms in a Fuel Cell System," Fuel Cells, 52018.

[16] K. Priya, T. Sudhakar Babu, K. Balasubramanian, K. Sathish Kumar, and N. Rajasekar, "A novel approach for fuel cell parameter estimation using simple Genetic Algorithm," Sustainable Energy Technologies and Assessments, vol. 12, pp. 46-52, 122015.

[17] W. Gong, Z. Cai, J. Yang, X. Li, and L. Jian, "Parameter identification of an SOFC model with an efficient, adaptive differential evolution algorithm," International Journal of Hydrogen Energy, vol. 39, no. 10, pp. 5083-5096, 32014.

[18] J. Na, M. N. Mahyuddin, G. Herrmann, and X. Ren, "Robust adaptive finite-time parameter estimation for linearly parameterized nonlinear systems," in Proceedings of the 32nd Chinese Control Conference, 7 2013, pp. 1735-1741.

[19] J. Na, M. N. Mahyuddin, G. Herrmann, X. Ren, and P. Barber, "Robust adaptive finite-time parameter estimation and control for robotic systems," International Journal of Robust and Nonlinear Control, vol. 25, no. 16, pp. 3045-3071, 112015.

[20] S. Strahl, A. Husar, P. Puleston, and J. Riera, "Performance Improvement by Temperature Control of an Open-Cathode PEM Fuel Cell System," Fuel Cells, vol. 14, no. 3, pp. 466-478, 62014.

[21] S. Strahl and R. Costa-Castelló, "Model-based analysis for the thermal management of open-cathode proton exchange membrane fuel cell systems concerning efficiency and stability," Journal of Process Control, vol. 47, pp. 201-212, 112016.

[22] A. L. Dicks and D. A. J. Rand, Fuel Cell Systems Explained. Chichester, UK: Wiley, 42018.

[23] M. d. Mathelin and R. Lozano, "Robust adaptive identification of slowly time-varying parameters with bounded disturbances," Automat$i c a$, vol. 35, no. 7, pp. 1291-1305, 71999. 